medición empleada, ya fuera la razón o la diferencia de las tasas (coeficiente de correlación de rangos de Spearman $=0,49$ ). De manera similar, la posición relativa de los países según la magnitud y la dirección de los cambios en los niveles de inequidad en el período de tiempo analizado varió también en dependencia del tipo de medición empleada. De gran importancia desde el punto de vista de la elaboración de políticas resultó el hecho de que la dirección de la tendencia observada mostró un cambio de signo (de aumento de la inequidad a la reducción) en cinco de los países (India, Namibia, Nepal, Perú y Vietnam) cuando se emplearon las dos mediciones estudiadas.

Esta es la primera comparación sistemática del uso de diferentes mediciones de la inequidad en salud, con herramientas estandarizadas, en un considerable número de países. El empleo indistinto de la razón de las tasas o de la diferencia de las tasas para medir la tendencia de los cambios en el nivel de inequidad generó diferencias sustanciales en el orden relativo que ocuparon los países analizados, tanto por la magnitud como por la dirección de los cambios. Estos resultados no se vieron afectados por factores de confusión - como el diseño del estudio, los métodos de obtención de los datos y los indicadores socioeconómicos, entre otros-y tienen importantes implicaciones para la elaboración de políticas, la atención sanitaria y la investigación.

A pesar de que no es posible hacer una recomendación categórica sobre las ventajas de medir la inequidad con métodos absolutos o relativos para comparar el efecto de las inequidades socioeconómicas en la salud, al analizar las inequidades y discutir las tasas se debe tomar en cuenta el método de medición utilizado, ya sea la razón de las tasas o la diferencia de las tasas, y el tipo de escala empleada, ya sea absoluta o relativa. (Moser K, Frost C, Leon DA. Comparing health inequalities across time and place - rate ratios and rate differences lead to different conclusions: analysis of cross-sectional data from 22 countries 1991-2001. Int J Epidemiol. 2007; 36:1285-91.)

\section{Estudio del Millón de Mujeres establece relación entre varios tipos de cáncer y el índice de masa corporal}

La prevalencia de la obesidad ha aumentado en el mundo. A pesar de que está ampliamente aceptado que el índice de masa corporal (IMC) está asociado directamente con el adenocarcinoma de esófago, el cáncer colorrectal, de endometrio y de riñón en la población general, y con el cáncer de mama en mujeres posmenopáusica, no se tienen datos exactos sobre la magnitud de este efecto y el papel que desempeña el IMC en el desarrollo de otros tipos de cáncer.

En este trabajo se investigó la relación entre la incidencia de y la mortalidad por 17 tipos/ubicaciones de cáncer y el IMC en las participantes en el denominado Estudio del Millón de Mujeres. Como parte de este estudio prospectivo de cohorte se realizó el seguimiento de 1,3 millones de mujeres de 50-64 años de edad del Reino Unido entre 1996 y 2001. El tiempo promedio de seguimiento fue de 5,4 años para la incidencia de cáncer y de 7,0 años para la mortalidad.

En el período de seguimiento se diagnosticaron 45037 casos de cáncer y ocurrieron 17203 muertes por esta causa. Se encontró una asociación significativa entre el aumento del IMC y el incremento en la incidencia de cáncer en 10 de las 17 ubicaciones específicas o tipos de cáncer estudiados: cáncer de endometrio (tendencia del riesgo relativo por 10 unidades $\left[\mathrm{tRR}_{10}\right]=2,89$; intervalo de confianza de 95\% [IC95\%]: 2,62 a 3,18), adenocarcinoma de esófago $\left(\mathrm{tRR}_{10}=2,38\right.$; IC95\%: 1,59 a 3,56), cáncer de riñón $\left(\mathrm{tRR}_{10}=1,53\right.$; IC95\%: 1,27 a 1,84), leucemia $\left(\mathrm{tRR}_{10}=1,50\right.$; IC95\%: 1,23 a 1,83), mielomas múltiples $\left(\mathrm{tRR}_{10}=1,31\right.$; IC95\%: 1,04 a 1,65$)$, cáncer de páncreas $\left(\mathrm{tRR}_{10}=1,24\right.$; IC95\%: 1,03 a $1,48)$, linfoma no Hodgkin $\left(\mathrm{tRR}_{10}=1,17\right.$; IC95\%: 1,03 a 1,34), cáncer de ovario $\left(\mathrm{tRR}_{10}=1,14 ; \mathrm{IC} 95 \%\right.$ : 1,03 a 1,27), todos los tipos de cáncer $\left(\operatorname{tRR}_{10}=1,12\right.$; IC95\%: 1,09 a 1,14), cáncer de mama en mujeres posmenopáusicas $\left(\mathrm{tRR}_{10}=1,40\right.$; $\mathrm{IC} 95 \%: 1,31$ a 1,49$)$ y cáncer colorrectal en mujeres premenopáusicas $\left(\mathrm{tRR}_{10}=1,61\right.$; IC95\%: 1,05 a 2,48). La asociación entre el IMC y la mortalidad por cáncer siguió un patrón similar al descrito para la incidencia.

El riesgo de cáncer de mama en mujeres premenopáusicas disminuyó a medida que aumentaba el IMC y aumentó con el IMC en las posmenopáusicas que no habían recibido tratamiento de sustitución hormonal. Estos datos demuestran que el estado de la mujer con relación a la menopausia constituye un factor clave en la relación entre el IMC y el riesgo de cáncer en mujeres, no solamente para los tipos de cáncer relacionados con las hormonas, como el cáncer de mama y de endometrio, sino también en otros tipos frecuentes de cáncer sin relación conocida con las hormonas. El efecto del IMC sobre el riesgo de melanomas malignos y cáncer colorrectal, de mama y de endometrio difiere significativamente según el estatus menopáusico de la mujer.

A pesar de la dificultad para lograr los estimados correspondientes a las mujeres que no han llegado a la menopausia con los datos disponibles, se concluyó que la proporción de casos de cáncer atribuibles al sobrepeso en mujeres premenopáusicas del Reino Unido puede ser menor que en mujeres posmenopáusicas, debido a la relación inversa 
observada en mujeres premenopáusicas entre el IMC y el riesgo de cáncer de mama, el más frecuente en estas mujeres. Según los resultados y los estimados actuales del IMC en las mujeres del Reino Unido, se estimó que 5\% de todos los casos de cáncer en mujeres posmenopáusicas de ese país están asociados con el sobrepeso o la obesidad (IMC $\geq 25)$ y que $4 \%$ están asociados con la obesidad (IMC $\geq 30$ ). En el caso del cáncer de endometrio y del adenocarcinoma de esófago, el IMC representa el principal factor de riesgo modificable.

Estos resultados constituyen la primera evidencia científica de la asociación entre el IMC y muchos de estos tipos de cáncer y el gran tamaño de la muestra le aporta una considerable robustez a estas conclusiones. En la Región de las Américas, donde el sobrepeso y la obesidad constituyen un grave y creciente problema, estos resultados deben tomarse muy en cuenta, tanto por los especialistas como por las autoridades de salud. (Reeves GK, Pirie K, Beral V, Green J, Spencer E, Bull D. Cancer incidence and mortality in relation to body mass index in the Million Women Study: cohort study. Br Med J. 2007; 335:1134.)

\section{Exposición al cadmio e hipertensión arterial en adultos fumadores y no fumadores}

El cadmio es un metal tóxico y carcinogénico de amplia distribución en la naturaleza. Las principales fuentes de exposición al cadmio en la población general son el humo del cigarro, el consumo de algunos alimentos y el aire ambiental, especialmente en zonas urbanas y en las proximidades de plantas industriales. La exposición al cadmio produce hipertensión en modelos animales, sin embargo los estudios epidemiológicos para determinar la asociación entre la exposición al cadmio ambiental y la tensión arterial en las personas no son concluyentes.

En este trabajo se investigó la asociación entre los niveles de cadmio en la sangre y la orina, por una parte, y la tensión arterial y la prevalencia de la hipertensión, por la otra, en una muestra representativa de los adultos de los Estados Unidos de América. El contenido de cadmio, tanto en la orina como en la sangre, es un marcador de la exposición prolongada o actual a ese metal, aunque los niveles en la sangre reflejan mejor la exposición reciente que los niveles en la orina.

Para este estudio se analizaron las concentraciones de cadmio en la sangre de 10991 personas y de cadmio en la orina de 3496 personas, del total de 15332 estadounidenses no institucionalizados de 20 años de edad o más participantes en la Encuesta Nacional de Salud y Nutrición (NHANES) de 1999-2004.

Las medias geométricas de la concentración de cadmio en la sangre y en la orina fueron 3,77 $\mathrm{nmol} / \mathrm{L}$ y 2,46 nmol/L, respectivamente. Después de ajustar por múltiples variables, las diferencias promedio de la tensión arterial sistólica entre el percentil 90 y el percentil 10 de la distribución de las concentraciones de cadmio en la sangre fue de 1,36 $\mathrm{mm} \mathrm{Hg}$ (intervalo de confianza de 95\% [IC95\%]: $-0,28$ a 3,00) y de cadmio en la orina de 1,68 $\mathrm{mm} \mathrm{Hg}$ (IC95\%: 0,57 a 2,78).

La asociación de las concentraciones de cadmio en la sangre y la orina y la tensión arterial fueron muy similares en hombres y mujeres, y no se observó ningún tipo de interacción significativa. Según su clasificación como fumadores, las diferencias promedio entre la tensión arterial sistólica y diastólica de los participantes ubicados en el percentil 90 por sus niveles de cadmio con respecto a los del percentil 10 fueron: 2,35 mm $\mathrm{Hg}$ y 3,27 mm $\mathrm{Hg}$, respectivamente, entre los que nunca fumaron; $1,69 \mathrm{~mm} \mathrm{Hg}$ y 1,55 mm Hg entre los ex fumadores; y $0,02 \mathrm{~mm} \mathrm{Hg}$ y $0,69 \mathrm{~mm} \mathrm{Hg}$ entre los fumadores activos. Las variaciones en la asociación entre los niveles de cadmio en la sangre y la tensión arterial sistólica y diastólica de los fumadores activos en comparación con los que nunca fumaron fueron significativas. Para los niveles de cadmio en la orina, esta asociación fue similar en todos los subgrupos de fumadores y no fumadores. No se encontró asociación entre el nivel de cadmio en la orina y la tensión arterial, o entre los niveles de cadmio en la sangre y la orina y la prevalencia de hipertensión.

A pesar del bajo nivel de exposición al cadmio observado en esta muestra representativa de la población adulta estadounidense, se encontró una ligera pero positiva asociación entre los niveles de cadmio en la sangre y la tensión arterial. Esta asociación fue más fuerte en las personas que nunca fumaron, intermedia en los ex fumadores y baja o nula en los fumadores. Deben emprenderse investigaciones similares en otros escenarios de la Región, donde la contaminación con cadmio puede ser mucho mayor. (Téllez-Plaza M, Navas-Acien A, Crainiceanu CM, Guallar E. Cadmium exposure and hypertension in the 1999-2004 National Health and Nutrition Examination Survey (NHANES). Environ Health Perspect. 2008;116(1). 